\title{
Using concept maps in cognitive treatment for children with developmental coordination disorder
}

\section{Tsameret Ricon}

Occupational Therapy Department. Faculty of Health \& Welfare Sciences, University of Haifa, Mount Carmel, Israel; tricon@univ.haifa.ac.il

Received 13 January 2010; revised 20 January 2010; accepted 21 January 2010.

\begin{abstract}
Children with Developmental Coordination Disorder (DCD) often seem to possess a narrow repertoire of cognitive strategies. In particular, they have difficulties in learning and internalizing the rules and strategies that other people intuitively use to approach common everyday problems. As a result, they often appear to have organizational, planning, memory and learning difficulties. The article proposes using a Concept Map (CM) as a visual strategy to facilitate interaction between a child with DCD, his/her family and therapist, as reflected in Client Centred and cognitive approaches. The CM is used as a method of assisting the child to identify, develop and utilize cognitive strategies in order to manage daily tasks effectively, as a tool in organizing his own therapy and in order to encourage participation. A demonstration of the concept mapping usefulness is brought by a case report. Further uses of concept mapping as a useful strategy within the framework of intervention remain to be studied.
\end{abstract}

Keywords: Concept Map; Cognitive Approach; Developmental Coordination Disorder; Organization Problems; Case Report

\section{INTRODUCTION}

Children with Developmental Coordination Disorder (DCD) experience problems with planning, ordering and then carrying out coordinated movements and tasks appropriate for their age. The use of cognitively-based interventions designed to help children with DCD to develop problem-solving strategies shows greater transfer to other areas of skill development than do traditional physical and occupational therapies [1]. This article aims to demonstrate the use of the concept map $(\mathrm{CM})$ as a cognitive strategy for treating children with DCD.

A concept map consists of a central word or concept surrounded by a few main ideas that relate to that word. It is a graphical two-dimensional display of concepts, connected by directed arcs encoding brief relationships (linking phrases) between pairs of concepts forming propositions.

Concept maps serves as tools for organizing and representing knowledge. They offer a method to represent information visually and therefore harness the power of our vision to understand complex information "at-aglance". We can assist the sequencing by which tasks are learned through attainment of progressively more explicit knowledge that can be anchored into developing conceptual frameworks.

Concept maps are, representation of concepts and their interrelationship, and are intended to represent the knowledge structures that humans store in their mind [2]. Concept maps are important when adopting a constructive view of learning. This view locates cognition and understanding within the individual [3], and is based on the theory that each of us develops mental schema that serve to inform future thinking or action [4]. It is thought that these schemas enable us to function with confidence in a complex environment, and it is these schemas that $\mathrm{CM}$ aims to represent diagrammatically.

In 1976, Novak [5] defined concept maps as a visual, organized representation of knowledge enhancing meaningful learning. Concept maps give structure to "the to-be-learned domain", including the before, during, and the after phases of action, thus playing an important role as an integrative organizer.

The use of cognitive-based approaches to enhance occupational performance (in the case of children with DCD, this is defined as their ability to successfully undertake their daily school, play, leisure and self care activities) has been a developing focus in contemporary occupational therapy literature [6]. In the early 1990s, Polatajko and colleagues [7] set out to develop a new 
approach to the treatment of children with DCD. Given the fact that children with DCD have difficulties in learning and generalizing motor skills, it seemed that the motor skills needed to be learned in more efficient way.

Meichenbaum [8,9] proposed that children could learn to regulate their behaviour by instructing themselves to identify a goal, develop a plan, enact the plan, and evaluate its success. In the Cognitive Orientation to daily Occupational Performance approach [10]; cognitive strategies are used to influence skill acquisition by children with occupational performance deficits. Generalization and transfer of skills is supported through the use of an executive or problem-solving strategy that trains the child to monitor his or her performance and selfevaluate the outcome. Domain-specific strategies form the bridge between the child's ability and skill level and help the child to develop appropriate motor plans.

\subsection{CM as a DCD Intervention Tool}

The behaviour of children with DCD often seems disorganized, presumably deriving from altered use of cognition, caused by otherwise biology (Figure 1) [11].

However we currently lack tools to directly assist children develop strategies that will enable them to become better organized at the cognitive level. In addition, to generalize and then transfer a learned strategy, the child must have knowledge of how, when and where to use that strategy [12]. Pressley and colleagues [12] recommend guided discovery learning as the optimal method for achieving transfer. CM, being a visual means of organizing cognition, can act precisely as such a learning method, and so assist children organize both their cognition and their behaviour, generalizing and transferring the learned strategies to other areas of their lives.
Intervention approaches have been mainly criticized for achieving only limited improvements for children with DCD in terms of generalized motor performance. An evaluation of the various techniques was performed by Mandich et al. [13], who found no difference, in terms of effectiveness, between Sensory Integrative (SI) therapy, physical education classes and perceptual motor treatment. A Meta analysis performed by Hen, Mayseles and Josman [14] analysed the mean effect size of the effectiveness of intervention approaches that had been reported in studies between 1987 and 2007, and found no significant differences in the effectiveness of the various approaches to treating children with DCD.

If we relate to DCD using Morton's model [11]—we see a biological defect that causes a cognitive conesquence that manifests as a behavioural impairment (see Figure 1). Similarly, DCD interventions should aim at the biological, consequence, or impairment levels (Figure 2).

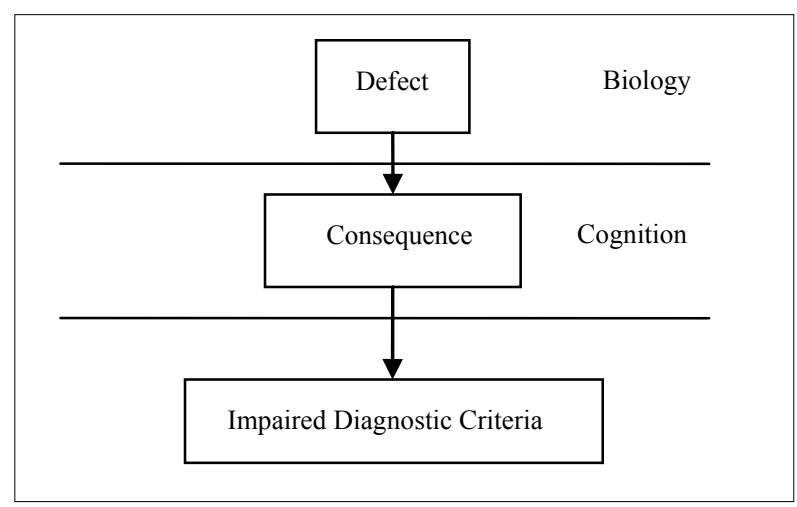

Figure 1. Morton's (2006) basic causal model.

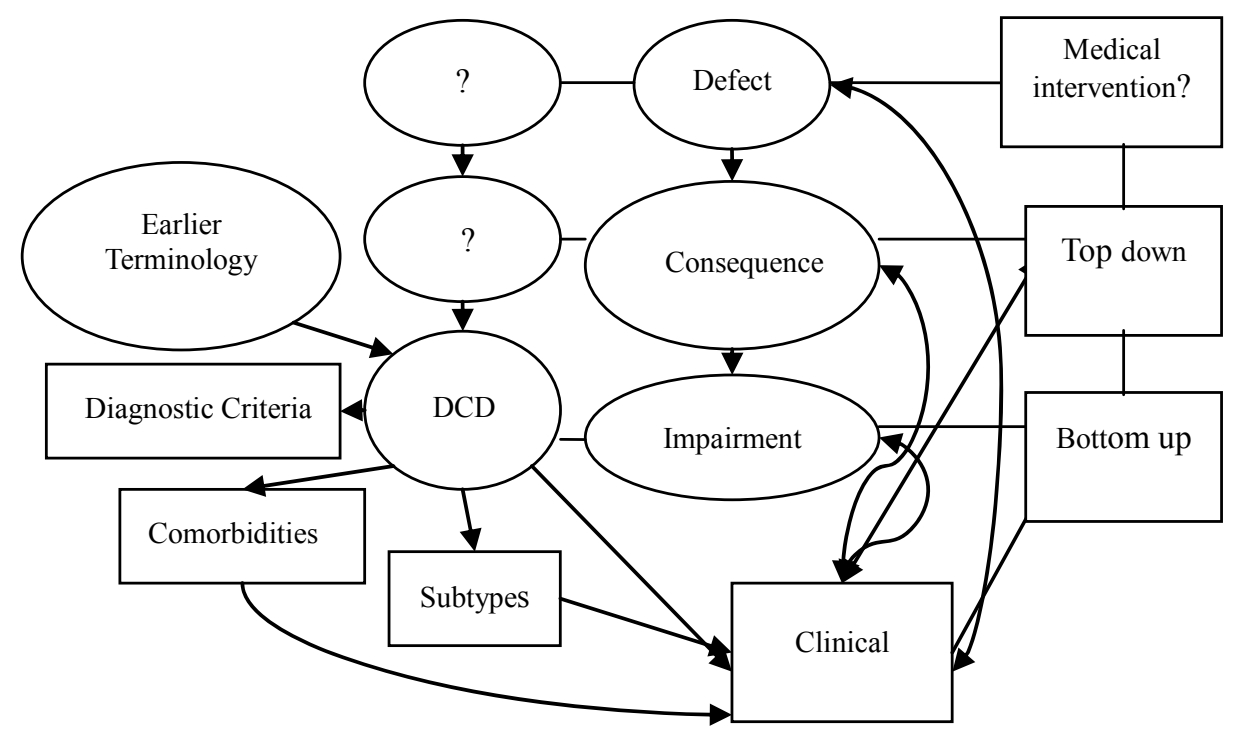

Figure 2. Concept Map of the Issues DCD Therapists must consider in making clinical Decisions. 
Neuromaturational, hierarchical theories consequently led to focus therapy on remediation underlying deficits with the expectation of subsequent improvement in motor performance. Other approaches define the objectives of intervention as facilitating skill acquisition, and therapy focuses on functional splinter skills. However, they have not been found to be effective in improving the functional ability of children with DCD [1]. These approaches need to be integrated into interventions aimed at higher levels.

Top down approaches focus on the role that the context of motor behaviour plays in organizing the motor system for performance. Contemporary theories propose that behaviour is self-organized and emerges from various subsystems, and emphasize a problem solving approach to motor skill acquisition. Top down approaches included task specific interventions, and cognitive treatments [1].

Cognitive theory literature has shown the use of strategies to facilitate performance. According to the Morton [11] model (Figure 1), the cognitive level is above the behavioural level of carrying out a task Cognitive strategies are in the conscious cognitive level and are controllable.

Feuerstein, Hoffman \& Miller [15] believed that chil- dren's cognition could be modified. Their cognitive deficiencies are observed either when the child approaches the task, thinks about the task or responds to it.

Consequently, Polatajko et al. [7] proposed a problem solving approach, which uses cognitive skills through verbal self guidance to improve the child's motor performance. In this top-down approach, the goal is the activity defined by the clients and objectives are: skill acquisition, transfer of skills across environments and tasks, and development of appropriate cognitive strategies [7]. Figure 3 illustrates where concept mapping fits within interventions aimed at the consequence (cognitive) level and within the broader DCD context.

This original pilot study suggests a step-by-step, new problem solving approach - using concept mapping. The visual presentation of organized linked ideas about any activity that is about to be done plays a role in facilitateing cognitive strategizing. This approach can be used with any age and with problems of any complexity by everyone: the child/parent/therapist/teacher/case manager and so forth. An example usage of CM to aid a child in achieving a desired skill is shown in Figures 4 and 5 .

Research into visual processing in children with DCD leads us to another controversial domain. Children with DCD experience difficulties in performance of all kinds

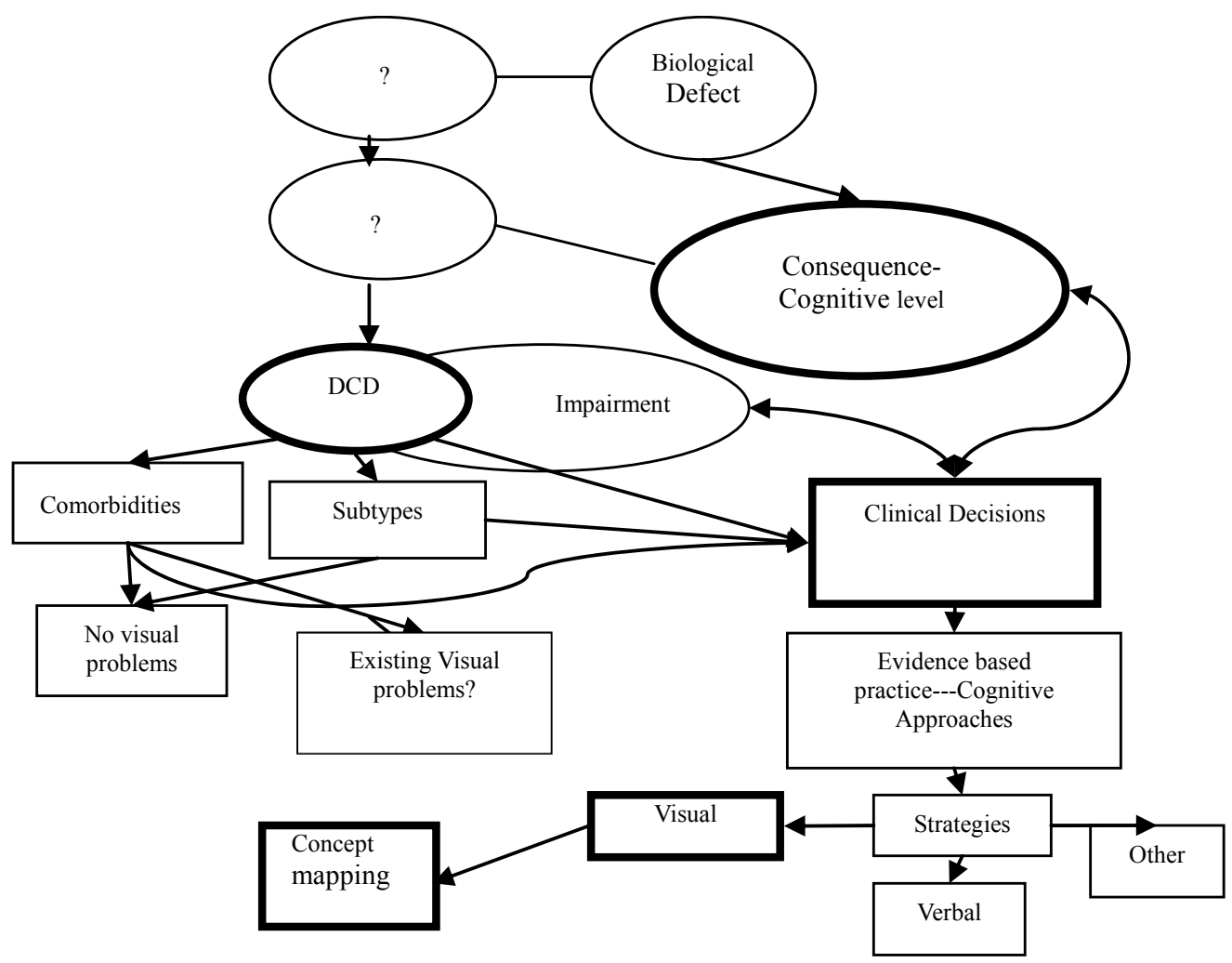

Figure 3. Clinical decisions deriving from the consequence level lead us to the use of cognitive approaches and concept mapping. 


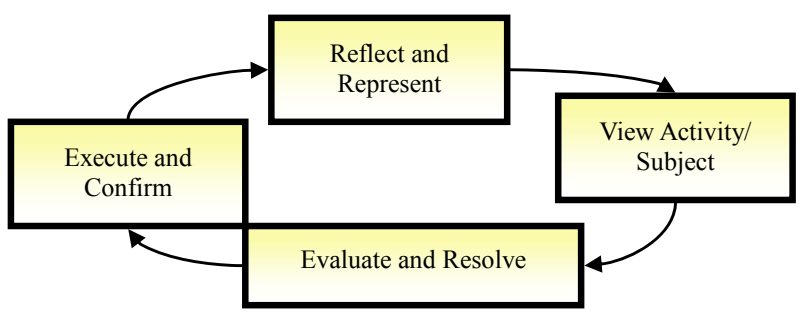

Figure 4. Phases of the constructed adoption of the visual strategy.

of motor skills, deficits in perceptual processes have often been assumed to underline these difficulties. Schoemaker and her colleagues [16] showed that children with DCD were not a homogenous group on the matter. They were as able as typical children to detect and match figures in a complex, confused background, and their most pronounced problems had motor nature. At this point the issue of co morbidity arises. Some studies that did show visual-perceptual problems failed to control for comorbidy and could not exclude the possibility of influence from other factors [17]. Another brief concept summary is presented in Figure 3.

Considering the overall picture with respect to DCD, and in light of the effectiveness of cognitive approaches, we suggest using the Concept Map as a cognitive visual tool in DCD intervention.

In the process of Concept Mapping, in parallel to the processes outline thus far, knowledge is anchored in conceptual frameworks that enable clients to develop more effective solutions to problems in occupational performance. Concept mapping can be used as a plan- ning tool prior to the activity, as an organizational strategy during the activity, and as a reflective activity that answers the needs of both the therapist and the child.

Using Concept Maps requires explication (making explicit what is normally implicit). The child becomes more aware of the required regulation of his/her learning processes in relation to task performance and the abilities to be acquired. Both explication and awareness contributes to the development of auto-monitoring techniques/strategies [4]. The constructed adoption of the visual strategy is demonstrated in Figure 4.

In the first node, the child is asked to "View the activity" in a preliminary stage that includes a graphic presentation of information. The activity could be any activity or even any subject, such as planning study for an exam or planning how to climb a ladder. In both cases, the aim is to arrive at implement able actions, bringing together all knowledge and previous experience. The second node is "Evaluate and resolve", and relates to the actual planning part. This is the process of building up a concept map in order to serve thinking/action and to enable functioning in a complex environment. The child is asked to elaborate on the problem verbally. $\mathrm{S} /$ he must write down or tell the therapist all the required steps of the activity or sub topics of the main subject, which require attention. Furthermore, the child must internalize and understand the relation between the sub-topics and the dynamic structure of the map. The therapist can serve as a mediator during this phase, to the extent required by children at different levels.

The next phase is "Execute and confirm", meaning

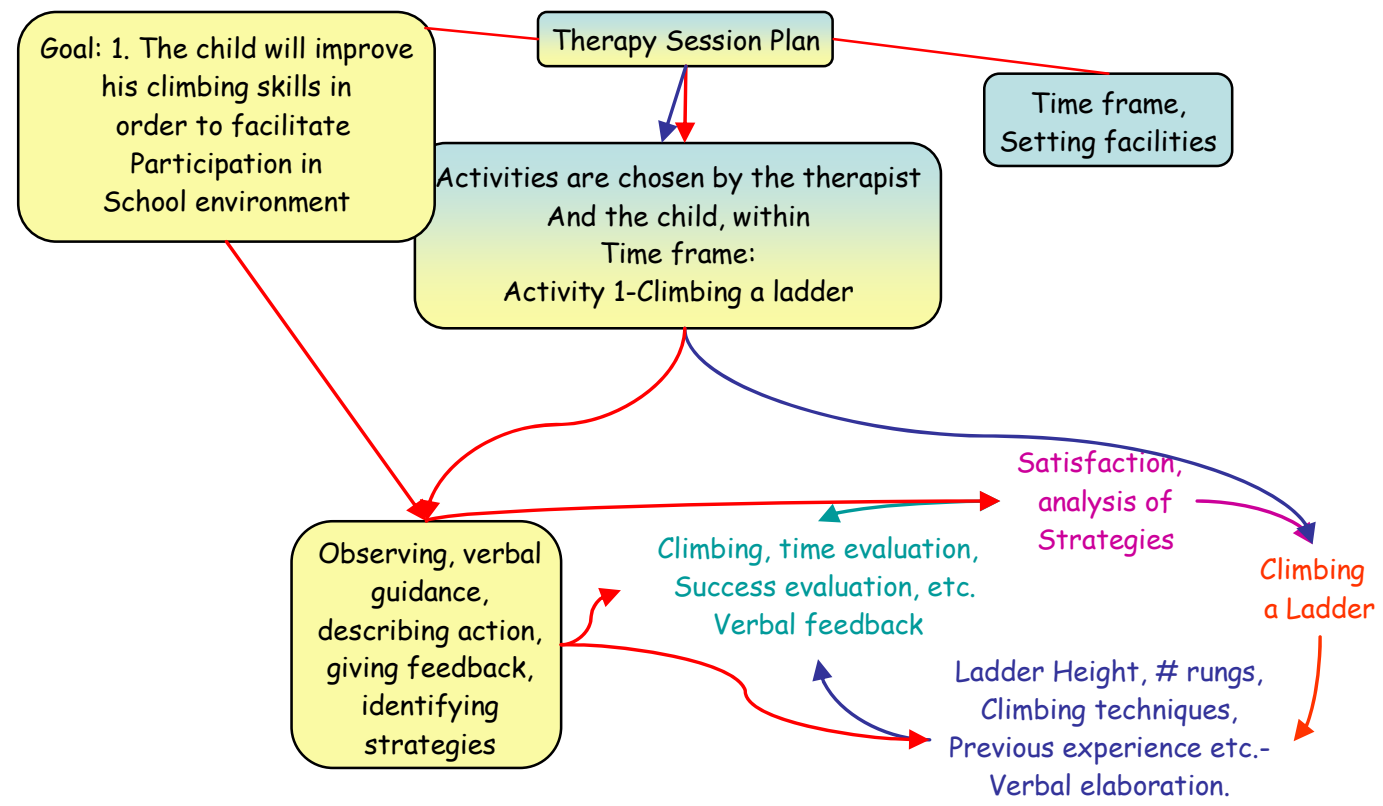

Figure 5. An example of concept map used in therapy. 
that the child executes as planned, and the therapist supports the child's actions with verbal guidance and reinforcement. During the last phase, "Reflect and represent", both the child and the therapist function as a "cognitive mirror". The child must reflect on his/her actions, organization and structure. With the assistance of the therapist, the child tries to manage cognitive resources more effectively and to establish/refine new/existing strategies.

An example of practical management of a $\mathrm{CM}$ is shown in Figure 5. The concept map must be a context related ("what are we going to do today") map. It has to have familiar content. We must ensure that the child recognizes the relationships between concepts (show $\mathrm{him} /$ her an example of a relationship, and let him point out a new one). And it is created by the child and therapist together, that is, it is "client cantered". After decideing with the child upon the activities to engage, the child elaborates on the activity verbally, make plans, draw them and so forth. Then execute and reflect on his performance.

The therapist has indirect control over the client's motivation in choosing to learn by attempting to incorporate new meanings into their prior knowledge, rather than simply memorizing the concept or plan. CM can function as an easy-to-use tool that enables the therapist to assess how the child is learning, where and how cognitive errors are occurring, and to undertake an overall evaluation of the learning strategies being taught.

\section{CASE STUDY}

\section{1. "Yoav"-An 8 Year Old Boy with DCD}

"Yoav" (the original name is kept confidential) an 8 year old boy was diagnosed by a physician as having DCD. He was referred to occupational therapy in order to relate to his organizational problems manifested in school and at home environments. Yoav has normal intelligence (IQ $\leq 85)$, no deficits in hearing and vision, and is right-handed. He and his parents had signed a parental consent and agreement to participate in therapy sessions using "concept map strategy", after they where explained about it. They also agreed to the presentation of this case report, anonymously.

Yoav did not get previous or other present cognitivebased treatment for his motor problems. Yoav was not diagnosed with any neurological disorder, physical or other sensory deficits.

\section{METHOD}

The tests that were administered during clinical intake included the Movement Assessment Battery for Children Test (M-ABC); [18] Movement Assessment Battery for Children (Manual). Sidcup, Kent, UK: The Psychological Corporation. Yoav scored less than the 15th percenttile, suggesting a DCD diagnosis.

\subsection{Pre- and Post-Measures Used to Evaluate Treatment}

In order to evaluate Changes in the tasks performance, identified by Yoav's perspective of himself, we used the Canadian Occupational Performance Measure (COPM) [19]. Canadian occupational performance measure. Toronto, Ont.: CAOT Publications A. The COPM is a semistructured interview designed to help clients identify problems in occupational performance. Yoav was asked to identify two major tasks out of five to be worked on during therapy. Using a 10-point scale, Yoav rated the tasks on perceived performance and satisfaction. The COPM was repeated after treatment to evaluate perceived change in performance and satisfaction.

Baseline data for the two tasks that Yoav defined were obtained prior to treatment. Performance baseline for each task was obtained for three repetitions of each of the two tasks. Yoav had chosen: 1. organizing his school bag 2. Making plans for a complex activity (such as studying for an exam, planning a trip). His performance seemed unorganized, unplanned and unsatisfying for him in terms of "end product" and self image.

\subsection{Treatment}

Over 10 individualized sessions, Yoav learned the concept mapping strategy and then applied it in performing better organization of trip planning and his school bag. Using the Concept mapping strategy, Yoav had learned to disassemble the task to its components and reorganize the task sequence and process through problem solving.

\subsection{Treatment Protocol}

The protocol of learning and implementing the concept map strategy in a chosen activity-planning a trip will be presented here : the overall sessions were built according to the phases of acquiring the visual strategy-"View the activity", "Evaluate and resolve", "Execute and confirm", "Reflect and represent".

Two first sessions were dedicated to learning the idea of concept-mapping strategy. On this stage we exercise different kinds of concept maps (family tree, human senses etc.) and made sure that Yoav could add new concepts or nodes and understood their relations, for example-we drew the basic family tree and ask him to add an extra horizontal node (in the same horizontal level of hierarchylike a brother or sister) and a vertical node (which is a different level in hierarchy like great 
grandmother above grandmother).

Session 3-5: gathering information for the trip plan to the desert. Organizing information becomes more important as the amount of information increases. Yoav and the therapist wrote down every trip planning component (equipment, maps, dates, tracks, food requirements, entertainment activities...) and made a graphic representation of them using computer flow charts (Figure 6).

Each time, adjustments were made. A new node or new connectors were added to the map. Parts that needed more information such as "tracks" were marked down and led to a new information quest. The quest usually yielded new nodes and new connectors, and usually new problems to solve (delays in driving hours, shortage of food and water supplies...).

Session 6 and 7: Defining responsibility domains according to the map: After finalizing map demands and domains, Yoav had to elaborate on it to his family members and give each member a responsibility domain. This presentation process strengthened his awareness and shown his broadening knowledge. Members of the family raised adequate questions.

This stage demonstrated the learning process-gathering knowledge-remembering, recognizing and identifying. Comprehension-interpreting, translating from one medium to another, describing in one's own words, or- ganization and problem solving-selection of facts and ideas, use of facts, analysis of rules and principles and Synthesis i.e. creating an original product (the concept map), forming a new ensemble using ideas that come from the analysis process.

Sessions 8-10: executing the plan and evaluating its success. On this phase, Yoav was requested to make value decisions about his plan; develop an opinion and judge decisions, and resolved ambiguity regarding the trip plan.

\subsection{Post Test}

After the completion of treatment, The COPM was readministered to Yoav. All activities were videotaped for future analysis.

\section{RESULTS}

Treatment effects were tested by comparing pre-test and post-test scores. The COPM performance and satisfaction ratings of Yoav were averaged across Yoav two goals, yielding an average performance rating and an average satisfaction rating. For both performance and satisfaction, improvements from pre-test to post-test were achieved (Table 1).

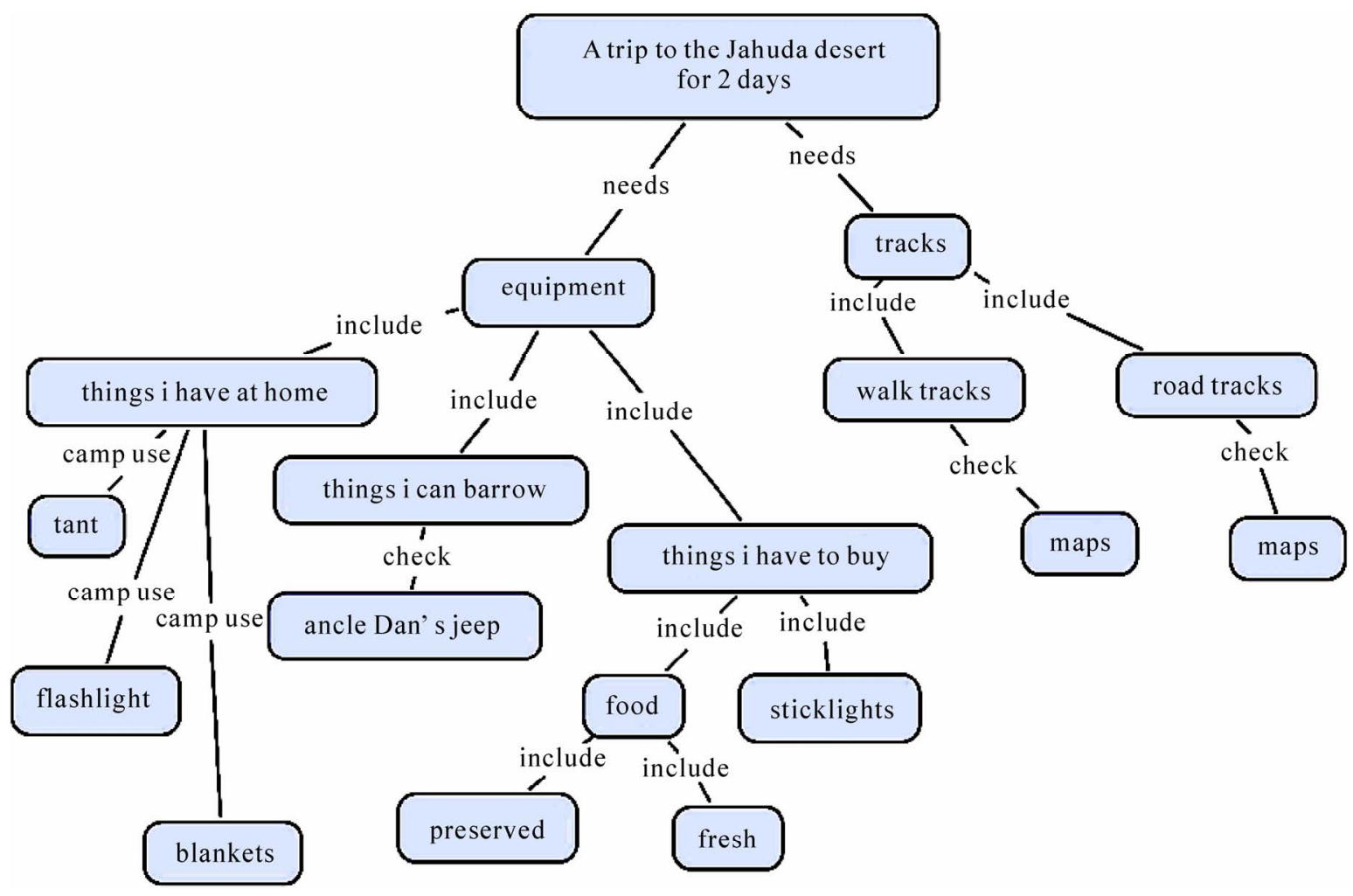

Figure 6. One of Yoav's concept maps in preparation and planning of the trip (translated from Hebrew). 
Table 1. COPM satisfaction and performance mean scores pre and post intervention.

\begin{tabular}{ccc}
\hline Score & Pre-test M & Post test M \\
\hline COPM performance & 4 & 8 \\
COPM satisfaction & 3 & 9 \\
\hline
\end{tabular}

\section{CONCLUSIONS}

The purpose of this article was to demonstrate the use of the concept map as a visual cognitive strategy in the treatment of children with DCD, and it's utility on as shown on a case report.

Similarly to other cognitive approaches, the use of concept mapping focuses directly on child-performance issues, and engages the child as an active problem solver and a significant partner in the therapy process. The results although limited to the case study and were done in a limited pre-post analysis, are encouraging with regard to the effectiveness of the concept mapping, and indicate that further uses of concept mapping as a useful visual strategy within the framework of intervention for children with DCD remain to be studied.

\section{REFERENCES}

[1] Miller, L., Polatajko, H., Missiuna C., Mandich, A.D. and Macnab, J. (2001) A pilot trial of a cognitive treatment for children with developmental coordination disorder. Human Movement Science, 20(1-2), 183-210.

[2] Jonassen, D., Beissner, K. and Yacci, M. (1993) Explicit methods for conveying structural knowledge through concept maps. Hillsdale, Erlbaum.

[3] Daley, B. (2004) Using Concept Maps with Adult Students in Higher Education. First International Conference on Concept Mapping, Universidad Pblica de Navarra, Spain.

[4] Bruillard, E. and Baron, G.L. (2000) Computer-based concept mapping: A review of a cognitive tool for students. In: Benzie, D. and Passey, D. Eds., Proceedings of Conference on Educational Uses of Information and Communication Technologies (ICEUT 2000), Publishing House of Electronics Industry (PHEI), Beijing, 331-338.

[5] Novak, J.D. (1976) Understanding the learning process and effectiveness of teaching methods in the classroom, laboratory, and field. Science. Education, 60(4), 493-512.

[6] Bernie, C. and Rodger, S. (2004). Cognitive strategy use in school-aged children with developmental coordination disorder. Physical and Occupational Therapy in Paediatrics, 24(4), 23-45.

[7] Polatajko, H.J., Mandich, A.D., Missiuna, C., Miller, L.T., Macnab, J.J., Malloy-Miller, T. and Kinsella, E.A. (2001) Cognitive orientation to daily occupational performance (CO-OP): Part III-the protocol in brief. Physical and Occupational Therapy in Paediatrics, 20(2-3), 107-123.

[8] Meichenbaum, D. (1977) Cognitive Behavior Modification, Plenum Press, New York.

[9] Meichenbaum, D. (1991) Cognitive Behavior Modification. Workshop presented at ChildandParent Research Inetitute Symposium, London, Ontario, Canada, London, Ontario Canada.

[10] Missiuna, C., Mandich, A.D., Polatajko, H.J. and MalloyMiller, T. (2001) Cognitive orientation to daily occupational performance (CO-OP): Part I-theoretical foundations. Physical and Occupational Therapy in Paediatrics, 20(2-3), 69-81.

[11] Morton, J. (2006) Understanding Developmental disorders: A Causal Modelling Approach. Black-well Publishing Ltd., Oxford.

[12] Pressley, M., Snyder, B.L. and Carglia-Bull, T. (1987). How can good strategy use be taught to children? Evaluation of six alternative approaches. In: Cormier, S. and Hagman, J. D. Eds., Transfer of Learning, Academic Press, 81-120.

[13] Mandich, A. D., Polatajko, H. J., Macnab, J. J. and Miller, L. T. (2001) Treatment of children with developmental coordination disorder: What is the evidence? Physical and Occupational Therapy in Pediatrics, 20(2-3), 51-68.

[14] Hen, L., Mayseless, O. and Josman, N. (on evaluation). The Effectiveness of Intervention Methods for Children with a Developmental Coordination Disorder (Children with DCD)-Meta Analysis, unpublished.

[15] Feuerstein, R., Hoffman, M. and Miller, R. (1980) Instrumental enrichment: An intervention program for cognitive modifiability. University Park Press, Baltimore.

[16] Schoemaker, M.M., Van der Wees, M., Flapper, B., Verheij-Jansen, N., Scholten-Jaegers, S. and Geuze, R.H. (2001) Perceptual skills of children with developmental coordination disorder. Human Movement Science, 20(1-2), 111-133.

[17] Van Waelvelde, H., De Weerdt, W., De Cock, P. and Smits-Engelsman, B.C. (2004). Association between visual perceptual deficits and motor deficits in children with developmental coordination disorder. Developmental Medicine \& Child Neurology, 46(10), 661-666.

[18] Henderson, S. and Sugden, D.A. (1992) Movement Assessment Battery for Children (Manual). The Psychological Corporation, Sidcup, Kent

[19] Law, M., Baptiste, S., Carswell, O.A., McColl, M.A., Polatajko, H. and Pollock, N. (1991) Canadian Occupational Performance Measure. CAOT Publications ACE, Toronto. 